\title{
Wave interactions with multiple-row curtainwall-pile breakwaters
}

\author{
Chang-Hwan Ji, Kyung-Duck Suh* \\ Department of Civil and Environmental Engineering, Seoul National University, 599 \\ Gwanangno, Gwanak-gu, Seoul 151-744, Republic of Korea
}

\begin{abstract}
In this study, a mathematical model has been developed that can compute various hydrodynamic characteristics of a multiple-row curtainwall-pile breakwater. To examine the validity of the developed model, laboratory experiments have been conducted for double- and triple-row breakwaters with various combinations of drafts of curtain walls, porosities between piles, and distances between rows. Comparisons between measurement and prediction show that the mathematical model adequately reproduces most of the important features of the experimental results. As a whole, the transmission coefficient decreases with an increase in relative water depth, whereas the reflection coefficient, normalized run-up and force exhibit an opposite trend in their variations. With fixed values of the draft of the curtain wall and the porosity of lower perforated part of the first row of a double-row breakwater, as these values of the second row increase and decrease, respectively, the transmission coefficient decreases, as expected. On the other hand, their effects on wave reflection, run-up, and wave force change with the relative depth. As for the distance between the rows, the transmission coefficient becomes a maximum when it is about one half of the wave length, suggesting that this condition should be avoided to achieve the advantage of the breakwater in reducing wave transmission. It is shown that for prototype breakwaters, on an average, the transmission coefficient would be smaller than 0.3 for wave periods less than $6.0 \mathrm{~s}$, and it would be about 0.45 even for the wave period of $9.0 \mathrm{~s}$, although there would be a variation depending on the geometry of the breakwater. It is also shown that wave transmission is significantly reduced by multiple-row breakwaters compared with a single-row breakwater, while the difference between double-row and triple-row breakwaters is marginal. Finally, engineering monograms are provided for double-row breakwaters to be used in practical engineering applications of the breakwaters.
\end{abstract}

\footnotetext{
${ }^{*}$ Corresponding author. Tel.: 822880 8760. E-mail address: kdsuh@ @nu.ac.kr (K.-D. Suh)
} 
Keywords: Breakwaters; Curtain walls; Laboratory tests; Mathematical models; Pile structures; Wave forces; Wave reflection; Wave run-up

\section{Introduction}

A curtainwall-pile breakwater (abbreviated as CPB hereinafter), the upper part of which is a curtain wall and the lower part consists of an array of vertical piles, may be a useful alternative to gravity-type breakwaters for protecting small craft harbors. In general, a $\mathrm{CPB}$ allows smaller construction costs and less environmental impacts compared with conventional gravity-type breakwaters. Suh et al. (2006) developed a mathematical model to predict wave transmission, reflection, run-up, and wave force acting on a CPB using the eigenfunction expansion method. They conducted large-scale laboratory experiments to examine the validity of the developed model, showing that the mathematical model adequately reproduces most of the important features of the experimental results. Later, Suh et al. (2007) modified the model to be used for circular piles instead of rectangular piles.

The CPB still gives large transmission for long-period waves. To reduce the wave transmission, the draft of the curtain wall must be increased or the porosity between the piles must be decreased. Then, however, the wave reflection and the wave force and moment acting on the breakwater may increase. Therefore, it is difficult to increase the draft or decrease the porosity beyond certain limits. A multiple-row CPB may be a solution for these problems. Furthermore, if we put a top plate between the rows, it should be useful to connect each row to keep the stability of the structure and to provide a space for fishing, walking, and maintenance of the breakwater, and so on.

Laju et al. (2007) have conducted numerical and experimental studies of the hydraulic characteristics of double-row CPB's. In this study, we extend the mathematical model of Suh et al. (2006) developed for a single row CPB to a multiple-row CPB to compute its various hydrodynamic characteristics. Hydraulic model experiments are carried out for a number of combinations of the drafts of the curtain walls, the porosity of lower perforated part, the distance between rows, and the number of rows. The experimental results are then compared with the predictions of the model to assess its 
performance. The average performance of double-row CPB's is examined based on the experimental results. The performance of a multiple-row CPB is compared against a single-row CPB. Finally, engineering monograms are developed to be used in practical engineering applications of CPB's.

\section{Mathematical model}

\subsection{Boundary value problem}

Let us consider the multiple-row CPB sketched in Fig. 1, in which $h=$ constant water depth in still water; $d_{j}=$ height of the $j$ th curtain wall below the still water level; $b_{j}$ $=$ thickness of the $j$ th wall. A Cartesian coordinate system $(x, z)$ is defined with the positive $x$ directing downwave from a point in front of the first wall and the vertical coordinate $z$ being measured vertically upwards from the still water line. The center of the $j$ th wall is located at $x=x_{j}$. The distance between the centers of two neighboring piles is denoted as $2 A_{j}$ and the width of the gap between the piles is $2 a_{j}$ so that the

porosity of the lower perforated part of the $j$ th wall is defined as $r_{j}=a_{j} / A_{j}$. A regular wave train with wave height $H_{i}$ is incident in the positive $x$-direction. The fluid domain is divided into $J+1$ regions by the $J$ walls. The upwave and downwave regions of the $j$ th wall are defined as $\Omega_{j-1}$ and $\Omega_{j}$, respectively.

Assuming incompressible fluid and irrotational flow motion, the velocity potential exists, which satisfies the Laplace equation. Linearizing the free-surface boundary conditions, the following boundary value problem is obtained for the velocity potential $\Phi_{j}(x, z, t)$ in each region:

$$
\begin{aligned}
& \frac{\partial^{2} \Phi_{j}}{\partial x^{2}}+\frac{\partial^{2} \Phi_{j}}{\partial z^{2}}=0 \\
& \frac{\partial \Phi_{j}}{\partial z}-\frac{\omega^{2}}{g} \Phi_{j}=0 \quad \text { at } \quad z=0 \\
& \frac{\partial \Phi_{j}}{\partial z}=0 \quad \text { at } \quad z=-h
\end{aligned}
$$


where $\omega=$ wave angular frequency; and $g=$ gravitational acceleration. Assuming periodic motion in time $t$, we can assume the solution to the above problem as

$$
\Phi_{j}(x, z, t)=\operatorname{Re}\left\{-\frac{i g H_{i}}{2 \omega} \frac{1}{\cosh (k h)} \phi_{j}(x, z) \exp (-i \omega t)\right\}
$$

where $i=\sqrt{-1}$; and the symbol Re represents the real part of a complex value. The wave number $k$ must satisfy the dispersion relationship, $\omega^{2}=g k \tanh (k h)$. The spatial variation of the velocity potential $\phi_{j}(x, z)$ should be determined in each region.

\subsection{Eigenfunction expansion method}

To solve the boundary value problem, we use the eigenfunction expansion method. We closely follow the method of Isaacson et al. (1998) and Suh et al. (2006), in which the velocity potential is expressed in a series of infinite number of solutions. The solutions to Eq. (1) satisfying the boundary conditions, Eqs. (2) and (3), in the regions of $\Omega_{0}$ and $\Omega_{J}$ are given by

$$
\begin{aligned}
& \phi_{0}=A_{00} \cos \left[\mu_{0}(h+z)\right] \exp \left[-\mu_{0}\left(x-x_{1}\right)\right]+\sum_{m=0}^{\infty} B_{0 m} \cos \left[\mu_{m}(h+z)\right] \exp \left[\mu_{m}\left(x-x_{1}\right)\right] \\
& \phi_{J}=\sum_{m=0}^{\infty} A_{J m} \cos \left[\mu_{m}(h+z)\right] \exp \left[-\mu_{m}\left(x-x_{J}\right)\right]
\end{aligned}
$$

respectively. Here $A_{j m}$ and $B_{j m}$ are the coefficients of the component waves propagating forward and backward, respectively. The first subscript $(j)$ indicates the row of the wall, while the second one $(m)$ indicates the wave component. The wave numbers $\mu_{m}$ are the solution to the dispersion relation, $\omega^{2}=-g \mu_{m} \tan \left(\mu_{m} h\right)$, which has an infinite discrete set of real roots $\pm \mu_{m}(m \geq 1)$ for non-propagating evanescent waves and a pair of imaginary roots $\mu_{0}= \pm i k$ for propagating waves. We take $\mu_{0}=-i k$ so that the propagating waves in Eqs. (5) and (6) correspond to the reflected and transmitted waves, respectively. We also take the positive roots for $m \geq 1$ so that the nonpropagating waves die out exponentially with the distance from the wall. Taking $A_{00}=1$, the first term on the right hand side of Eq. (5) represents the incident wave potential, and $\left|B_{00}\right|$ and $\left|A_{J 0}\right|$ are the reflection and transmission coefficients, respectively. On the other hand, the solutions in the regions from $\Omega_{1}$ to $\Omega_{J-1}$ are given by 


$$
\phi_{j}=\sum_{m=0}^{\infty}\left\{A_{j m} \exp \left[-\mu_{m}\left(x-x_{j}\right)\right]+B_{j m} \exp \left[\mu_{m}\left(x-x_{j+1}\right)\right]\right\} \cos \left[\mu_{m}(h+z)\right], \quad j=1,2, \cdots, J-1
$$

We assume that the length scale of the flow near the wall is much smaller than the wave length, so that the wall has no thickness mathematically and the three-dimensional feature near the wall does not significantly affect the two-dimensional solutions far from the wall. Then $\phi_{j-1}(x, z)$ and $\phi_{j}(x, z)$ must satisfy the following matching conditions at $x=x_{j}$ :

$$
\begin{aligned}
& \frac{\partial \phi_{j-1}}{\partial x}=\frac{\partial \phi_{j}}{\partial x}=0 \quad \text { for } \quad-d_{j} \leq z \leq 0, \quad x=x_{j} \\
& \frac{\partial \phi_{j-1}}{\partial x}=\frac{\partial \phi_{j}}{\partial x}=i G_{j}\left(\phi_{j-1}-\phi_{j}\right) \quad \text { for } \quad-h \leq z \leq-d_{j}, \quad x=x_{j}
\end{aligned}
$$

The first matching condition describes that the horizontal velocities vanish on both sides of the upper curtain wall. The second one for the lower perforated part of the wall describes that the horizontal velocities in the two regions must be same at the wall and that the horizontal velocity at the opening is proportional to the pressure difference across the wall. The proportional constant $G_{j}$, often called permeability parameter, is in general complex. There are several ways to express the constant $G_{j}$. Suh et al. (2006) have used the method of Mei et al. (1974) with the energy dissipation coefficient given by Kim (1998). They found that the energy dissipation coefficient of Kim (1998) is not applicable for small values of $k h$ because it goes to infinity as $k h$ goes to zero. In the present study, the method of Sollitt and Cross (1972) is followed and $G_{j}$ is expressed by

$$
G_{j}=\frac{r_{j}}{b_{j}\left(f_{j}-i s_{j}\right)}
$$

where $f_{j}=$ friction coefficient; and $s_{j}=$ inertia coefficient given by 


$$
s_{j}=1+C_{m}\left(\frac{1-r_{j}}{r_{j}}\right)
$$

where $C_{m}=$ added mass coefficient. These coefficients have been estimated on the basis of a best fit between measurement and prediction. Recently, Li et al. (2006) proposed an empirical equation for estimating $f$ in terms of $b / h$, along with the use of $C_{m}=0$, which predicts monotonic decrease of $f$ with $b / h$ from 9.2 to 4.5 in the range of validity of $0.0094 \leq b / h \leq 0.05$. They also recommended using $f=2.0$ if $b / h \geq 0.1$. The value of $b / h$ in the present study is 0.06 , which is located neither in the range of validity of their equation nor in the range of $b / h \geq 0.1$. The value of $f$ for $b / h=0.06$ calculated by extrapolating their equation is 1.66 , which is smaller than the constant value 2.0 recommended for $b / h \geq 0.1$. Therefore, in the present study, $f_{j}=2.0$ and $C_{m}=0$ were used. Isaacson et al. (1998) also used these values, which were shown to make their numerical results agree well with their experimental results for vertical slotted barriers.

Now Eqs. (5) to (7) satisfy the free surface boundary condition in Eq. (2) and the bottom boundary condition in Eq. (3). Also, they automatically satisfy the requirement that the horizontal velocities must be matched at the wall. In order to calculate the unknown coefficients $A_{j m}$ 's and $B_{j m}$ 's, we use the matching conditions at the walls, i.e., Eqs. (8) and (9). First, for $x=x_{1}$, Eqs. (5) and (7) are substituted into Eqs. (8) and (9), respectively. Multiplying each resulting equation by $\cos \left[\mu_{n}(h+z)\right]$, integrating with respect to $z$ over the appropriate domain of $z$ (i.e., $z=-d_{1}$ to 0 , or $z=-h$ to $-d_{1}$ ), and finally adding them, we obtain

$$
\begin{aligned}
& \sum_{m=0}^{\infty}\left[\left\{\mu_{m} f_{m n}\left(-d_{1}, 0\right)+\left(\mu_{m}-i G_{1}\right) f_{m n}\left(-h,-d_{1}\right)\right\} B_{0 m}\right. \\
& \left.\left.\quad+i G_{1} f_{m n}\left(-h,-d_{1}\right) A_{1 m}+i G_{1} \exp \left(-\mu_{m} \Delta x_{1}\right) f_{m n}\left(-h,-d_{1}\right)\right\} B_{1 m}\right] \\
& =\mu_{0} A_{00}\left\{f_{0 n}\left(-d_{1}, 0\right)+f_{0 n}\left(-h,-d_{1}\right)\right\}+i G_{1} A_{00} f_{0 n}\left(-h,-d_{1}\right) \text { for } n=0,1,2, \cdots, \infty
\end{aligned}
$$

and 


$$
\begin{aligned}
& \sum_{m=0}^{\infty}\left[\mu_{m}\left\{f_{m n}\left(-d_{1}, 0\right)+f_{m n}\left(-h,-d_{1}\right)\right\} B_{0 m}+\mu_{m}\left\{f_{m n}\left(-d_{1}, 0\right)+f_{m n}\left(-h,-d_{1}\right)\right\} A_{1 m}\right. \\
& \left.\quad-\mu_{m} \exp \left(-\mu_{m} \Delta x_{1}\right)\left\{f_{m n}\left(-d_{1}, 0\right)+f_{m n}\left(-h,-d_{1}\right)\right\} B_{1 m}\right] \\
& =\mu_{0} A_{00}\left\{f_{0 n}\left(-d_{1}, 0\right)+f_{0 n}\left(-h,-d_{1}\right)\right\} \quad \text { for } n=0,1,2, \cdots, \infty
\end{aligned}
$$

where

$$
\Delta x_{j}=x_{j+1}-x_{j}
$$

and

$$
\begin{aligned}
f_{m n}(p, q) & =\int_{p}^{q} \cos \left[\mu_{m}(h+z)\right] \cos \left[\mu_{n}(h+z)\right] d z \\
& = \begin{cases}\frac{1}{2}\left[\frac{\sin \left[\left(\mu_{m}+\mu_{n}\right)(h+z)\right]}{\mu_{m}+\mu_{n}}+\frac{\sin \left[\left(\mu_{m}-\mu_{n}\right)(h+z)\right]}{\mu_{m}-\mu_{n}}\right]_{p}^{q} & \text { for } m \neq n \\
\frac{1}{4 \mu_{\mathrm{m}}}\left[2 \mu_{m}(h+z)+\sin \left[2 \mu_{m}(h+z)\right]\right]_{p}^{q} & \text { for } m=n\end{cases}
\end{aligned}
$$

For $x=x_{j}(j=2$ to $J-1)$, Eq. (7) is substituted into Eqs. (8) and (9), respectively. Again, multiplying each resulting equation by $\cos \left[\mu_{n}(h+z)\right]$, integrating with respect to $z$ over the appropriate domain of $z$, and finally adding them, we obtain

$$
\begin{aligned}
\sum_{m=0}^{\infty} & {\left[\exp \left(-\mu_{m} \Delta x_{j-1}\right)\left\{-\mu_{m} f_{m n}\left(-d_{j}, 0\right)+\mu_{m} f_{m n}\left(-h,-d_{1}\right)+i G_{j} f_{m n}\left(-h,-d_{j}\right)\right\} A_{j-1, m}\right.} \\
& +\left\{\mu_{m} f_{m n}\left(-d_{j}, 0\right)-\mu_{m} f_{m n}\left(-h,-d_{j}\right)+i G_{j} f_{m n}\left(-h,-d_{j}\right)\right\} B_{j-1, m} \\
\left.-i G_{j} f_{m n}\left(-h,-d_{j}\right) A_{j m}-i G_{j} \exp \left(\mu_{m} \Delta x_{j}\right) f_{m n}\left(-h,-d_{j}\right) B_{j m}\right]=0 & \text { for } n=0,1,2, \cdots, \infty
\end{aligned}
$$

and

$$
\begin{aligned}
& \sum_{m=0}^{\infty}\left[\mu_{m} \exp \left(-\mu_{m} \Delta x_{j-1}\right)\left\{f_{m n}\left(-d_{j}, 0\right)+f_{m n}\left(-h,-d_{j}\right)\right\} A_{j-1, m}\right. \\
& \quad-\mu_{m}\left\{f_{m n}\left(-d_{j}, 0\right)+f_{m n}\left(-h,-d_{j}\right)\right\} B_{j-1, m}-\mu_{m}\left\{f_{m n}\left(-d_{j}, 0\right)+f_{m n}\left(-h,-d_{j}\right)\right\} A_{j m} \\
& \left.+\mu_{m} \exp \left(-\mu_{m} \Delta x_{j}\right)\left\{f_{m n}\left(-d_{j}, 0\right)+f_{m n}\left(-h,-d_{j}\right)\right\} B_{j m}\right]=0 \\
& \\
& \quad \text { for } n=0,1,2, \cdots, \infty
\end{aligned}
$$


Finally, for $x=x_{J}$, Eqs. (6) and (7) are substituted into Eqs. (8) and (9), respectively. Using the similar procedure as above, we obtain

$$
\begin{aligned}
\sum_{m=0}^{\infty} & {\left[\exp \left(-\mu_{m} \Delta x_{J-1}\right)\left\{-\mu_{m} f_{m n}\left(-d_{J}, 0\right)+\mu_{m} f_{m n}\left(-h,-d_{J}\right)+i G_{J} f_{m n}\left(-h,-d_{J}\right)\right\} A_{J-1, m}\right.} \\
& +\left\{\mu_{m} f_{m n}\left(-d_{J}, 0\right)-\mu_{m} f_{m n}\left(-h,-d_{J}\right)+i G_{J} f_{m n}\left(-h,-d_{J}\right)\right\} B_{J-1, m} \\
& \left.-i G_{J} f_{m n}\left(-h,-d_{J}\right) A_{J m}\right]=0 \quad \text { for } n=0,1,2, \cdots, \infty
\end{aligned}
$$

and

$$
\begin{aligned}
\sum_{m=0}^{\infty} & {\left[\mu_{m} \exp \left(-\mu_{m} \Delta x_{J-1}\right)\left\{f_{m n}\left(-d_{J}, 0\right)+f_{m n}\left(-h,-d_{J}\right)\right\} A_{J-1, m}\right.} \\
& -\mu_{m}\left\{f_{m n}\left(-d_{J}, 0\right)+f_{m n}\left(-h,-d_{J}\right)\right\} B_{J-1, m} \\
& \left.-\mu_{m}\left\{f_{m n}\left(-d_{J}, 0\right)+f_{m n}\left(-h,-d_{J}\right)\right\} A_{J m}\right]=0 \quad \text { for } n=0,1,2, \cdots, \infty
\end{aligned}
$$

If we take $M$ wave modes (i.e., one progressive and $M-1$ evanescent modes), the number of unknown coefficients is 2JM . Eqs. (12), (13), and (16) to (19) give $2 J M$ equations, which can be solved for the unknown coefficients.

\subsection{Engineering wave properties}

Once the wave potentials are calculated, we can obtain various engineering wave properties. The reflection and transmission coefficients are given by

$$
C_{r}=\left|B_{00}\right|
$$

and

$$
C_{t}=\left|A_{J 0}\right|
$$

respectively. The wave run-up on the upwave side of the first row of the breakwater is given by

$$
R_{u}=\frac{H_{i}}{2} \frac{1}{\cosh (k h)}\left|A_{00} \cos \left(\mu_{0} h\right)+\sum_{m=0}^{\infty} B_{0 m} \cos \left(\mu_{m} h\right)\right|
$$


In the limiting case of a full-depth impermeable vertical wall $\left(d_{1} \rightarrow h\right.$ or $\left.r_{1} \rightarrow 0\right)$, $B_{0 m}=0$ for all $m \geq 1$ and $B_{00}=A_{00}=1$ so that $C_{r}=1.0$ and $R_{u}=H_{i}$ as expected.

The wave force on each wall can be calculated by integrating the wave pressure acting on both upwave and downwave sides of the wall. The maximum horizontal wave force $F_{\max }$ per unit width of the first wall of breakwater is given by

$$
\begin{aligned}
F_{\max }= & \frac{\rho g H_{i}}{2} \frac{1}{\cosh (k h)} \mid\left\{\frac{A_{00}}{\mu_{0}} \sin \left(\mu_{0} h\right)+\sum_{m=0}^{\infty} \frac{1}{\mu_{m}}\left\{B_{0 m}-A_{1 m}-B_{1 m} \exp \left[\mu_{m}\left(x_{1}-x_{2}\right)\right]\right\} \sin \left(\mu_{m} h\right)\right\} \\
& -r_{1}\left\{\frac{A_{00}}{\mu_{0}} \sin \left[\mu_{0}\left(h-d_{1}\right)\right]+\sum_{m=0}^{\infty} \frac{1}{\mu_{m}}\left\{B_{0 m}-A_{1 m}-B_{1 m} \exp \left[\mu_{m}\left(x_{1}-x_{2}\right)\right]\right\} \sin \left[\mu_{m}\left(h-d_{1}\right)\right]\right\} \mid \\
+ & \frac{\rho g H_{i}^{2}}{8} \frac{1}{\cosh ^{2}(k h)}\left\{\left(A_{00} \cos \left(\mu_{0} h\right)+\sum_{m=0}^{\infty} B_{0 m} \cos \left(\mu_{m} h\right)\right)^{2}\right. \\
& \left.-\left(\sum_{m=0}^{\infty}\left\{A_{1 m}+B_{1 m} \exp \left[\mu_{m}\left(x_{1}-x_{2}\right)\right]\right\} \cos \left(\mu_{m} h\right)\right)^{2}\right\}
\end{aligned}
$$

where $\rho=$ density of fluid. The second term on the right-hand side represents the second-order force contribution of the wave crest regions on the upwave and downwave sides of the wall (Dean and Dalrymple 1991). Without this term, in the limiting case of a full-depth impermeable vertical wall $\left(d_{1} \rightarrow h\right.$ or $\left.r_{1} \rightarrow 0\right), B_{0 m}=0$ for all $m \geq 1$, $B_{00}=A_{00}=1$, and $A_{1 m}=B_{1 m}=0$ for all $m \geq 0$, so that the preceding equation becomes

$$
F_{\text {max }}^{s}=\frac{\rho g H_{i}}{k} \mathrm{tank}(h)
$$

where the superscript $s$ stands for the standing wave in front of an impermeable vertical wall.

\section{Laboratory experiment}

Experiments were carried out in the wave flume at the Coastal Engineering Laboratory of Seoul National University. Fig. 2 shows the arrangement of the model breakwater and wave gauges. The flume was 30-m long, 0.6-m wide, and 1-m deep. It was equipped 
with a piston-type wave generator at one end, and a wave-absorbing slope at the other. The first wall of the breakwater model was placed at a distance of $18.5 \mathrm{~m}$ from the wave maker. Water surface displacements were measured with parallel-wire resistance-type wave gauges.

All experiments were conducted at a water depth of $0.5 \mathrm{~m}$. Most experiments were carried out for double-row breakwaters, while a few cases of triple-row were also tested. Square piles with side lengths of $3 \mathrm{~cm}$ were used, with $2 a=1.0,2.0$ and $3.0 \mathrm{~cm}$, for which $2 A=4.0,5.0$, and $6.0 \mathrm{~cm}$, respectively, so that the porosity of the lower perforated wall was $0.25,0.4$, and 0.5 , respectively. Three different drafts of the upper curtain wall were used; 25, 30, and $37.5 \mathrm{~cm}$. Different combinations of these porosities and drafts were used with different distances between walls. In all the cases, the porosity of the lower perforated wall and the draft of the upper curtain wall of the first wall were $r_{1}=0.5$ and $d_{1}=25 \mathrm{~cm}$, respectively. The walls were high enough above the water level to prevent wave overtopping.

Six different wave periods $(T=1.0,1.2,1.4,1.6,1.8,2.0 \mathrm{~s})$ were used with specified wave heights corresponding to a constant wave steepness, $H / L=0.03$. The measured values of the incident wave heights and periods for double-row breakwaters are given in Table 1, along with the reflection and transmission coefficients, and run-up and wave forces on the first wall.

To measure the incident, reflected, and transmitted waves, and wave run-up at front and rear sides of the first wall, seven wave gauges were installed as shown in Fig. 2. Wave measurements were made for $75 \mathrm{~s}$ at a sampling rate of $20 \mathrm{~Hz}$ immediately after the initiation of wave generation. The free surface displacements measured at WG2 to WG4 in Fig. 2 were used to separate the incident and reflected waves using the technique developed by Suh et al. (2001). The incident waves measured at WG1 was used to crosscheck the incident waves obtained by the separation. The transmitted waves were measured using one wave gage at WG7 assuming that the wave reflection from the downwave slope is negligible. Previous observations indicated reflection coefficients from the slope smaller than 0.1 for the wave periods used in these tests. The wave run-up at the front and rear sides of the first wall were measured by the wave gauges at WG5 and WG6.

Fourteen pressure sensors were used to measure the wave pressure acting on the wall; eight on the front side $(-5,-2.5,0,2.5,5,20,28,40 \mathrm{~cm}$ below still 
water level) and six on the rear side $(0,2.5,5,20,28,40 \mathrm{~cm}$ below still water level) of the wall. The pressure measurements were only made for the first wall where the greatest wave force is expected. The duration and sampling rate of pressure measurements were the same as those of wave measurements. Determining the instantaneous elevations of water surface by the run-up wave gages at WG5 and WG6, the wave pressures are vertically integrated from sea bed to water surface to obtain the total forces acting on each side of the wall. In the lower part of the wall consisting of piles, the pressure is multiplied by $1-r_{1}$ because the pressure acts only on the piles there. The maximum difference between the front and rear forces is the maximum wave force acting on the wall. The maximum wave force slightly changes with the waves even though regular waves are used. In the present study, we used the average value of the first three established waves regardless wave period. Fig. 3 shows an example of temporal variations of water surface elevations at the front and rear sides of the first wall (i.e., $\eta_{5}$ and $\eta_{6}$ ) and their difference as well as the total wave force, $F_{f}-F_{r}$, where $F_{f}$ and $F_{r}$ respectively are the wave forces acting on the front and rear faces of the wall. In this case, $x_{2}-x_{1}=0.5 h, d_{2}=0.5 h, r_{2}=0.5, T=1.0 \mathrm{~s}$, and $H=4.04 \mathrm{~cm}$ were used. The surface elevations $\eta_{5}$ and $\eta_{6}$ are almost out of phase so that the difference between them is almost in phase with the front surface elevation $\eta_{5}$. Consequently, the maximum total force occurs when the difference between front and rear surface elevations reaches maximum. At this time, the wave crests still just attack at the first wall.

\section{Comparison with experimental results}

In this section, the mathematical model results are compared with the experimental results. The effects of various parameters upon the hydrodynamic characteristics of the breakwater are also discussed where appropriate. The number of terms used in the eigenfunction expansion method was 50, which was found to give accurate results over the range of values presented here. In all the results presented hereafter, the draft of the curtain wall and the porosity of lower perforated part of the first row are fixed as $0.5 \mathrm{~h}$ 
and 0.5 , respectively. The parameters whose effects are examined are the draft of the curtain wall and the porosity of lower perforated part of the second row and the distance between the first and second rows. Most of the results are for double-row CPB's, but some results for triple-row CPB's are also presented.

Fig. 4 shows a comparison between measurement and prediction of reflection and transmission coefficients and wave run-up and maximum wave force on the first row of the breakwater as functions of $k h$ for different drafts of the curtain wall of the second row (i.e., $d_{2}$ ). The porosity of lower perforated part of the second row was $r_{2}=0.5$ and the distance between the first and second rows was $x_{2}-x_{1}=h$. The wave run-up was normalized with respect to the incident wave height, and the wave force was normalized with respect to that on a full-depth impermeable vertical wall, $F_{\max }^{s}$, given by Eq. (24). In general, the mathematical model adequately reproduces most of the important features of the experimental results, even though the reflection and transmission coefficients are somewhat under-predicted and over-predicted, respectively, for relatively long waves (i.e., in the range of $k h$ less than 1.0). The transmission coefficient decreases as $d_{2}$ increases, as expected. It is interesting to note that the effect of $d_{2}$ upon the reflection coefficient and wave run-up is different depending on the relative depth. In the range of $k h$ between approximately 0.7 and 1.1, both the reflection coefficient and wave run-up decrease as $d_{2}$ increases, while the opposite is shown in other values of $k h$. For the wave force, the effect of $d_{2}$ becomes opposite at $k h$ of about 0.7 , though its effect is not great. The normalized run-up is greater than 1.0 for shorter waves even though the lower part of the breakwater is perforated, which is known to be 1.0 for a full-depth impermeable vertical wall. This is due to the inclusion of the evanescent waves. Without these, the normalized run-up would be always smaller than 1.0 and converge to 1.0 as $k h$ increases.

The wave run-up and maximum wave force are shown only for the first row of the breakwater in Fig. 4, since these values on the second row are in general smaller than those on the first row. To confirm this, comparisons for these two properties between the first and second rows using the mathematical model results are shown in Fig. 5. $d_{2}=0.5 h$ was used with other structural parameters being the same as those used for Fig. 4. For both properties, the value on the second row is much smaller than that on the first row for $k h \geq 1.5$, while they are almost equal in magnitude for smaller $k h$.

Fig. 6 shows a comparison similar to Fig. 4 for different porosities between piles of 
the second row (i.e., $r_{2}$ ). The draft of the curtain wall of the second row was $d_{2}=0.5 \mathrm{~h}$ and the distance between the first and second rows was $x_{2}-x_{1}=h$. The decrease of $r_{2}$ has the same effect as the increase of $d_{2}$. Therefore, the overall trends with decreasing $r_{2}$ are very similar to those with increasing $d_{2}$ as shown in Fig. 4. However, it is seen that the increase of $d_{2}$ affects the hydrodynamic characteristics of the CPB more than the decrease of $r_{2}$ when the ratio of the opening area among piles to the total area is kept the same. Note that this ratio is the same for the case of $d_{2}=0.75 h$ in Fig. 4 and that of $r_{2}=0.25$ in Fig. 6 .

Fig. 7 shows a similar comparison for different distances between the first and second rows (i.e., $\Delta x_{1}=x_{2}-x_{1}$ ). The draft of the curtain wall and the porosity of lower perforated part of the second row were $d_{2}=0.5 h$ and $r_{2}=0.5$, respectively. It is noticeable that the depression points of the hydrodynamic characteristic values occur at different values of $k h$ depending on $\Delta x_{1}$. The depression points of the reflection coefficient and wave run-up occur at almost same value of $k h$, shifting toward smaller $k h$ as $\Delta x_{1}$ increases. It is also observed that another depression point appears at a larger value of $k h$ as $\Delta x_{1}$ increases. The transmission coefficient and maximum wave force show opposite variation each other with respect to $k h$ so that the former shows a peaked value when the latter shows a depression. Again the locations of these peaked or depressed values shift toward smaller $k h$ as $\Delta x_{1}$ increases.

In order to examine the effect of the distance between the rows in more detail, the variations of the reflection and transmission coefficients are plotted as functions of the relative spacing, $\Delta x_{1} / L$, for $k h$ varying from $0.2 \pi$ to $\pi$ in steps of $0.2 \pi$ in Fig. 8 . Again the draft of the curtain wall and the porosity of lower perforated part of the second row were $d_{2}=0.5 h$ and $r_{2}=0.5$, respectively. Both the reflection and transmission coefficients oscillate with $\Delta x_{1} / L$, showing that the locations of their maximum and minimum change depending on the relative water depth. Irrespective of the relative water depth, however, the transmission coefficient is found to be a maximum when $\Delta x_{1} / L$ is between 0.5 and 0.6. The results suggest that the distance between the two rows of a double-row CPB should not be near one half of the wave length to achieve its advantage in attenuating the incident waves.

Fig. 9 shows comparisons between measurement and prediction for three different cases of triple-row breakwaters. The structural parameters of the second and third rows 
are the same as those of the first row, i.e., $d_{1}=d_{2}=d_{3}=0.5 h$ and $r_{1}=r_{2}=r_{3}=0.5$. The distance between the first and third rows was fixed as $x_{3}-x_{1}=2 h$ and the location of the second row was changed so that $\Delta x_{1}$ becomes $0.5 h, 1.0 h$ and $1.5 h$. The overall agreement between measurement and prediction is acceptable. It is interesting to note that the calculated transmission coefficients are the same for the cases of $\Delta x_{1}=0.5 h$ and $\Delta x_{1}=1.5 h$ so that the two curves are indistinguishable in the figure. Without showing the results, we mention that the transmission coefficients of a triple-row breakwater are calculated to be the same when the values of $\Delta x_{1}$ and $\Delta x_{2}$ are switched with a fixed value of $x_{3}-x_{1}$.

\section{Average performance of double-row CPB's}

In general, the reflection and transmission coefficients of a permeable breakwater like a CPB are primarily dependent upon the wave period. In order to roughly examine the performance of a CPB depending on wave periods, all the experimental results of double-row CPB's were converted to prototype values and plotted as functions of $g T^{2} / h$ in Fig. 10. Assuming the length scale of the experiment to be 1:20, the water depth, wave height, and wave period in prototype are $10 \mathrm{~m}, 0.9$ to $2.5 \mathrm{~m}$, and 4.5 to $8.9 \mathrm{~s}$, respectively. Even for the same wave period, the reflection and transmission coefficients vary greatly because the draft of the curtain wall, the porosity of lower perforated part, and the distance between rows are different one another. As a whole, the reflection and transmission coefficients decrease and increase, respectively, as the wave period increases. On an average, the transmission coefficient is smaller than 0.3 for wave periods less than $6.0 \mathrm{~s}$, and it is about 0.45 even for the wave period of $9.0 \mathrm{~s}$.

\section{Comparison with single-row CPB}

A multiple-row CPB would in general allow less wave transmission than a single-row CPB. It also has several advantages as discussed in the introduction. However, it necessitates a greater construction cost than a single-row CPB. It may be interesting to compare the performance of a multiple-row CPB against a single-row CPB, especially in 
the ability to reduce wave transmission.

Fig. 11 shows comparisons among single-, double-, and triple-row CPB's. We used $d=0.5 h$ and $r=0.5$ for all the CPB's and a constant spacing $\Delta x=1.0 h$ in doubleand triple-row CPB's. Wave reflection and run-up show relatively small difference in spite of oscillatory behaviors for multiple-row CPB's, because these characteristics are primarily influenced by the first row. Wave transmission and wave force on the first row show greater differences. Wave transmission is significantly reduced by the multiple-row CPB's, especially for shorter waves (i.e. $k h>1.0$ ), while wave force increases for a multiple-row CPB. On the other hand, the difference between double-row and triple-row CPB's is not significant. Therefore, a triple-row CPB would not be recommended unless reducing wave transmission is extremely important.

\section{Engineering monograms}

It may be useful to provide engineers some design-oriented monograms for practical engineering applications of CPB's. We only provide monograms for double-row CPB's since increasing rows does not significantly improve the performance of the breakwater. Figs. 12 to 16 show variations of reflection and transmission coefficients and maximum wave run-up and force on the front row of the breakwater as functions of $k h$ for different $d_{1} / h, d_{2} / h, r_{1}, r_{2}$, and $\Delta x / h$, respectively. While one of these variables changes as indicated in the figures, the remaining variables are fixed as $d_{1} / h=0.5$, $d_{2} / h=0.5, r_{1}=0.5, r_{2}=0.5$, and $\Delta x / h=1.0$.

Investigation of Figs. 12 to 16 shows that the hydrodynamic characteristics of CPB's are most significantly influenced by the change of the draft of the curtain wall of the front row, while the least influential parameter is the porosity of lower perforated part of the second row. It is interesting to note that the wave transmission coefficient is the same in Figs. 12 and 13 and in Figs. 14 and 15, respectively. This means that switching the first and second rows does not make any difference in wave transmission. Other hydrodynamic characteristics, however, are changed by switching the rows.

\section{Conclusions}


By extending the work of Suh et al. (2006), we developed a mathematical model to calculate various hydrodynamic characteristics of a multiple-row $\mathrm{CPB}$. In order to examine the validity of the developed model, laboratory experiments were undertaken that involved regular waves of various heights and periods impinging upon CPB's having various drafts of curtain walls, porosities between piles, and distances between rows.

Comparisons between measurement and prediction showed that the mathematical model was able to adequately reproduce most of the important features of the experimental results. As a whole, the transmission coefficient decreased with the relative water depth, whereas the reflection coefficient and normalized run-up and wave force increased with the relative depth, although all of them exhibited oscillatory behavior with the relative depth. When the draft of the curtain wall and the porosity of lower perforated part of the first row of a double-row CPB are fixed, as these values of the second row increased and decreased, respectively, the transmission coefficient decreased, as expected. On the other hand, their effects on wave reflection, run-up, and wave force changed with the relative depth. As for the distance between the rows, the transmission coefficient was found to be a maximum when it is about one half of the wave length, suggesting that this condition should be avoided to achieve the advantage of a doublerow $\mathrm{CPB}$ in reducing wave transmission.

It was shown that for prototype CPB's in $10 \mathrm{~m}$ water depth, on an average, the transmission coefficient would be smaller than 0.3 for wave periods less than $6.0 \mathrm{~s}$, and it would be about 0.45 even for the wave period of $9.0 \mathrm{~s}$, although there would be a variation depending on the geometry of the breakwater. It was also shown that wave transmission is significantly reduced by multiple-row CPB's compared with a single-row $\mathrm{CPB}$, whereas the difference between double-row and triple-row CPB's is marginal. Therefore, a triple-row $\mathrm{CPB}$ would not be recommended unless reducing wave transmission is extremely important. Finally, engineering monograms were provided for double-row CPB's to be used in practical engineering applications of CPB's. The most influential parameter on the performance of a double-row CPB was found to be the curtain-wall draft of the first row, while the least influential one the porosity of lower perforated part of the second row. It was found that switching the first and second rows does not make any difference in wave transmission, while other hydrodynamic characteristics are changed by switching the rows. For real use of a CPB in engineering 
practice, investigation for irregular waves may be necessary in the future.

\section{Acknowledgments}

This work was supported by the Korea Sea Grant Program and the Brain Korea 21 Project. This work was conducted at the Engineering Research Institute of Seoul National University.

\section{References}

Dean, R.G., Dalrymple, R.A., 1991. Water Wave Mechanics for Engineers and Scientists. World Scientific, Singapore.

Isaacson, M., Premasiri, S., Yang, G., 1998. Wave interactions with vertical slotted barrier. Journal of Waterway, Port, Coast. and Ocean Engineering 124, 118-126.

Kim, B.H., 1998. Interactions of waves, seabed and structures. PhD dissertation, Seoul National University.

Laju, K., Sundar, V., Sundaravadivelu, R., 2007. Studies on pile supported double skirt breakwater models. The Journal of Ocean Technology 2, 33-53.

Li, Y., Liu, Y., Teng, B., 2006. Porous effect parameter of thin permeable plates. Coastal Engineering Journal 48, 309-336.

Mei, C.C., Liu, P.L.-F., Ippen, A.T., 1974. Quadratic loss and scattering of long waves. Journal of Waterway, Harbors, and Coastal Engineering Division 100, 217-239.

Sollitt, C.K., Cross, R.H., 1972. Wave transmission through permeable breakwaters. In: Proceedings of 13th International Conference on Coastal Engineering, pp. 1827-1846.

Suh, K.-D., Jung, H.Y., Pyun, C.K., 2007. Wave reflection and transmission by curtainwall-pile breakwaters using circular piles. Ocean Engineering 34, 2100-2106.

Suh, K.D., Park, W.S., Park, B.S., 2001. Separation of incident and reflected waves in wave-current flumes. Coastal Engineering 43, 149-159.

Suh, K.-D., Shin, S., Cox, D.T. 2006. Hydrodynamic characteristics of pile-supported vertical wall breakwaters. Journal of Waterway, Port, Coast. and Ocean Engineering 132, 83-96. 
Table 1. Summary of experimental results for double-row breakwaters

\begin{tabular}{c|r|c|c|r|c|c|c|c}
\hline \hline$\frac{x_{2}-x_{1}}{h}$ & \multicolumn{1}{c|}{$\frac{d_{2}}{h}$} & $r_{2}$ & $\begin{array}{c}T \\
(\mathrm{~s})\end{array}$ & \multicolumn{1}{|c|}{$H_{i}$} & \multicolumn{1}{c|}{$\mathrm{C}_{r}$} & $C_{t}$ & $\frac{R_{u}}{H_{i}}$ & $\frac{F_{\max }}{F_{\max }^{s}}$ \\
\hline 1.0 & 0.5 & 0.5 & 1.0 & 4.05 & 0.93 & 0.07 & 1.03 & 0.88 \\
1.0 & 0.5 & 0.5 & 1.2 & 5.53 & 0.67 & 0.21 & 0.99 & 0.84 \\
1.0 & 0.5 & 0.5 & 1.4 & 6.66 & 0.44 & 0.39 & 0.79 & 0.80 \\
1.0 & 0.5 & 0.5 & 1.6 & 8.21 & 0.32 & 0.46 & 0.67 & 0.60 \\
1.0 & 0.5 & 0.5 & 1.8 & 9.18 & 0.29 & 0.59 & 0.64 & 0.60 \\
1.0 & 0.5 & 0.5 & 2.0 & 10.42 & 0.36 & 0.57 & 0.64 & 0.51 \\
1.0 & 0.6 & 0.5 & 1.0 & 4.10 & 0.93 & 0.07 & 1.04 & 0.94 \\
1.0 & 0.6 & 0.5 & 1.2 & 5.55 & 0.70 & 0.15 & 1.00 & 0.99 \\
1.0 & 0.6 & 0.5 & 1.4 & 6.53 & 0.40 & 0.35 & 0.80 & 0.61 \\
1.0 & 0.6 & 0.5 & 1.6 & 8.12 & 0.32 & 0.39 & 0.60 & 0.45 \\
1.0 & 0.6 & 0.5 & 1.8 & 9.10 & 0.39 & 0.46 & 0.58 & 0.45 \\
1.0 & 0.6 & 0.5 & 2.0 & 10.46 & 0.33 & 0.51 & 0.61 & 0.36 \\
1.0 & 0.75 & 0.5 & 1.0 & 4.05 & 0.93 & 0.07 & 1.04 & 0.89 \\
1.0 & 0.75 & 0.5 & 1.2 & 5.38 & 0.65 & 0.13 & 0.98 & 0.84 \\
1.0 & 0.75 & 0.5 & 1.4 & 6.60 & 0.45 & 0.24 & 0.81 & 0.81 \\
1.0 & 0.75 & 0.5 & 1.6 & 7.98 & 0.27 & 0.30 & 0.54 & 0.73 \\
1.0 & 0.75 & 0.5 & 1.8 & 9.14 & 0.39 & 0.36 & 0.50 & 0.65 \\
1.0 & 0.75 & 0.5 & 2.0 & 10.47 & 0.29 & 0.38 & 0.54 & 0.60 \\
1.0 & 0.5 & 0.25 & 1.0 & 4.03 & 0.92 & 0.06 & 1.10 & 0.91 \\
1.0 & 0.5 & 0.25 & 1.2 & 5.50 & 0.69 & 0.16 & 0.97 & 0.82 \\
1.0 & 0.5 & 0.25 & 1.4 & 6.70 & 0.44 & 0.31 & 0.81 & 0.78 \\
1.0 & 0.5 & 0.25 & 1.6 & 8.12 & 0.29 & 0.35 & 0.69 & 0.71 \\
1.0 & 0.5 & 0.25 & 1.8 & 9.29 & 0.29 & 0.38 & 0.58 & 0.66 \\
1.0 & 0.5 & 0.25 & 2.0 & 10.26 & 0.28 & 0.44 & 0.61 & 0.57 \\
1.0 & 0.5 & 0.4 & 1.0 & 3.96 & 0.91 & 0.09 & 1.09 & 0.91 \\
1.0 & 0.5 & 0.4 & 1.2 & 5.53 & 0.68 & 0.18 & 0.93 & 0.99 \\
1.0 & 0.5 & 0.4 & 1.4 & 6.67 & 0.44 & 0.37 & 0.80 & 0.61 \\
1.0 & 0.5 & 0.4 & 1.6 & 8.13 & 0.34 & 0.41 & 0.69 & 0.45 \\
1.0 & 0.5 & 0.4 & 1.8 & 9.19 & 0.39 & 0.48 & 0.61 & 0.45 \\
1.0 & 0.5 & 0.4 & 2.0 & 10.20 & 0.38 & 0.54 & 0.63 & 0.36 \\
0.5 & 0.5 & 0.5 & 1.0 & 4.04 & 0.88 & 0.06 & 1.04 & 0.98 \\
0.5 & 0.5 & 0.5 & 1.2 & 5.39 & 0.56 & 0.29 & 0.95 & 0.89 \\
0.5 & 0.5 & 0.5 & 1.4 & 6.32 & 0.38 & 0.41 & 0.73 & 0.54 \\
0.5 & 0.5 & 0.5 & 1.6 & 7.89 & 0.46 & 0.56 & 0.75 & 0.39 \\
0.5 & 0.5 & 0.5 & 1.8 & 9.54 & 0.44 & 0.57 & 0.76 & 0.42 \\
0.5 & 0.5 & 0.5 & 2.0 & 11.67 & 0.42 & 0.68 & 0.71 & 0.35 \\
\hline \hline
\end{tabular}


Table 1. Continued

\begin{tabular}{c|c|c|c|r|c|c|c|c}
\hline \hline$\frac{x_{2}-x_{1}}{h}$ & $\frac{d_{2}}{h}$ & $r_{2}$ & $\begin{array}{c}T \\
(\mathrm{~s})\end{array}$ & $\begin{array}{c}H_{i} \\
(\mathrm{~cm})\end{array}$ & $C_{r}$ & $C_{t}$ & $\frac{R_{u}}{H_{i}}$ & $\frac{F_{\max }}{F_{\max }^{s}}$ \\
\hline 1.5 & 0.5 & 0.5 & 1.0 & 4.00 & 0.93 & 0.18 & 1.00 & 0.49 \\
1.5 & 0.5 & 0.5 & 1.2 & 5.49 & 0.71 & 0.19 & 1.03 & 0.73 \\
1.5 & 0.5 & 0.5 & 1.4 & 6.56 & 0.55 & 0.30 & 0.93 & 0.75 \\
1.5 & 0.5 & 0.5 & 1.6 & 8.29 & 0.39 & 0.37 & 0.79 & 0.72 \\
1.5 & 0.5 & 0.5 & 1.8 & 9.22 & 0.29 & 0.41 & 0.66 & 0.69 \\
1.5 & 0.5 & 0.5 & 2.0 & 10.11 & 0.21 & 0.46 & 0.61 & 0.56 \\
2.0 & 0.5 & 0.5 & 1.0 & 4.06 & 0.83 & 0.15 & 1.04 & 0.99 \\
2.0 & 0.5 & 0.5 & 1.2 & 5.48 & 0.82 & 0.30 & 1.00 & 0.47 \\
2.0 & 0.5 & 0.5 & 1.4 & 6.78 & 0.70 & 0.34 & 0.89 & 0.61 \\
2.0 & 0.5 & 0.5 & 1.6 & 8.28 & 0.49 & 0.37 & 0.76 & 0.69 \\
2.0 & 0.5 & 0.5 & 1.8 & 9.24 & 0.37 & 0.39 & 0.67 & 0.75 \\
2.0 & 0.5 & 0.5 & 2.0 & 10.12 & 0.18 & 0.51 & 0.52 & 0.63 \\
1.0 & 0.6 & 0.4 & 1.0 & 3.92 & 0.93 & 0.07 & 1.06 & 0.92 \\
1.0 & 0.6 & 0.4 & 1.2 & 5.37 & 0.69 & 0.14 & 0.99 & 0.81 \\
1.0 & 0.6 & 0.4 & 1.4 & 7.33 & 0.43 & 0.30 & 0.63 & 0.81 \\
1.0 & 0.6 & 0.4 & 1.6 & 8.26 & 0.31 & 0.34 & 0.58 & 0.97 \\
1.0 & 0.6 & 0.4 & 1.8 & 9.20 & 0.37 & 0.46 & 0.54 & 0.64 \\
1.0 & 0.6 & 0.4 & 2.0 & 10.25 & 0.34 & 0.46 & 0.49 & 0.53 \\
1.0 & 0.75 & 0.25 & 1.0 & 3.96 & 0.92 & 0.09 & 1.07 & 0.85 \\
1.0 & 0.75 & 0.25 & 1.2 & 5.30 & 0.71 & 0.12 & 0.99 & 0.84 \\
1.0 & 0.75 & 0.25 & 1.4 & 6.42 & 0.46 & 0.20 & 0.79 & 0.81 \\
1.0 & 0.75 & 0.25 & 1.6 & 8.06 & 0.29 & 0.24 & 0.55 & 0.77 \\
1.0 & 0.75 & 0.25 & 1.8 & 9.20 & 0.24 & 0.27 & 0.46 & 0.72 \\
1.0 & 0.75 & 0.25 & 2.0 & 10.73 & 0.28 & 0.30 & 0.42 & 0.64 \\
\hline \hline
\end{tabular}




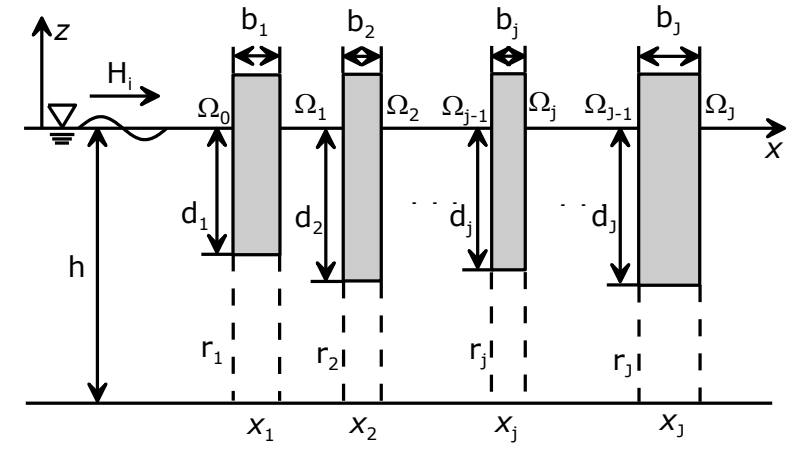

(a)

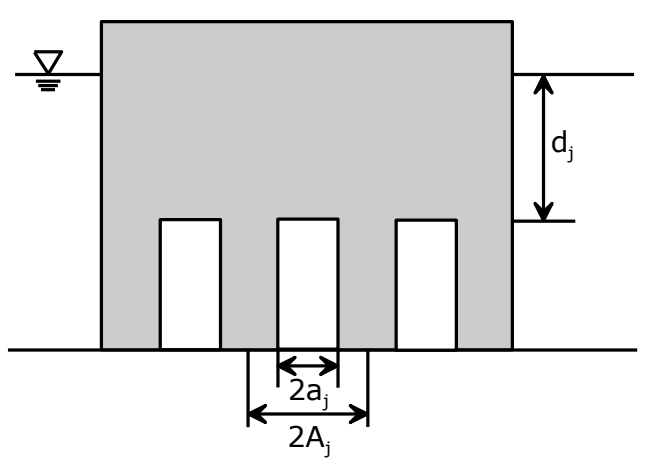

(b)

Fig. 1. Definition sketch: (a) side view; and (b) front view of $j$-th row 


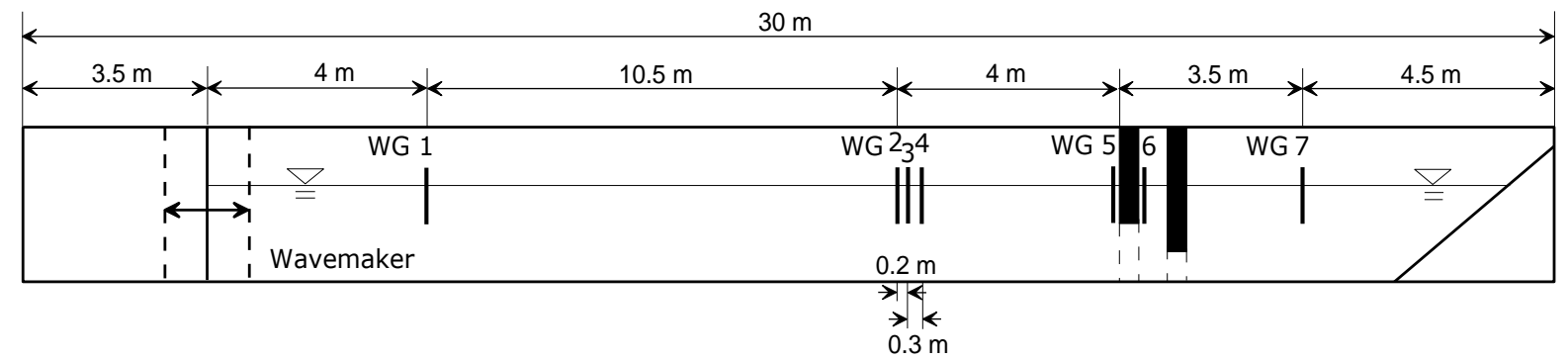

Fig. 2. Sketch of wave flume and experimental setup. WG=wave gauge 


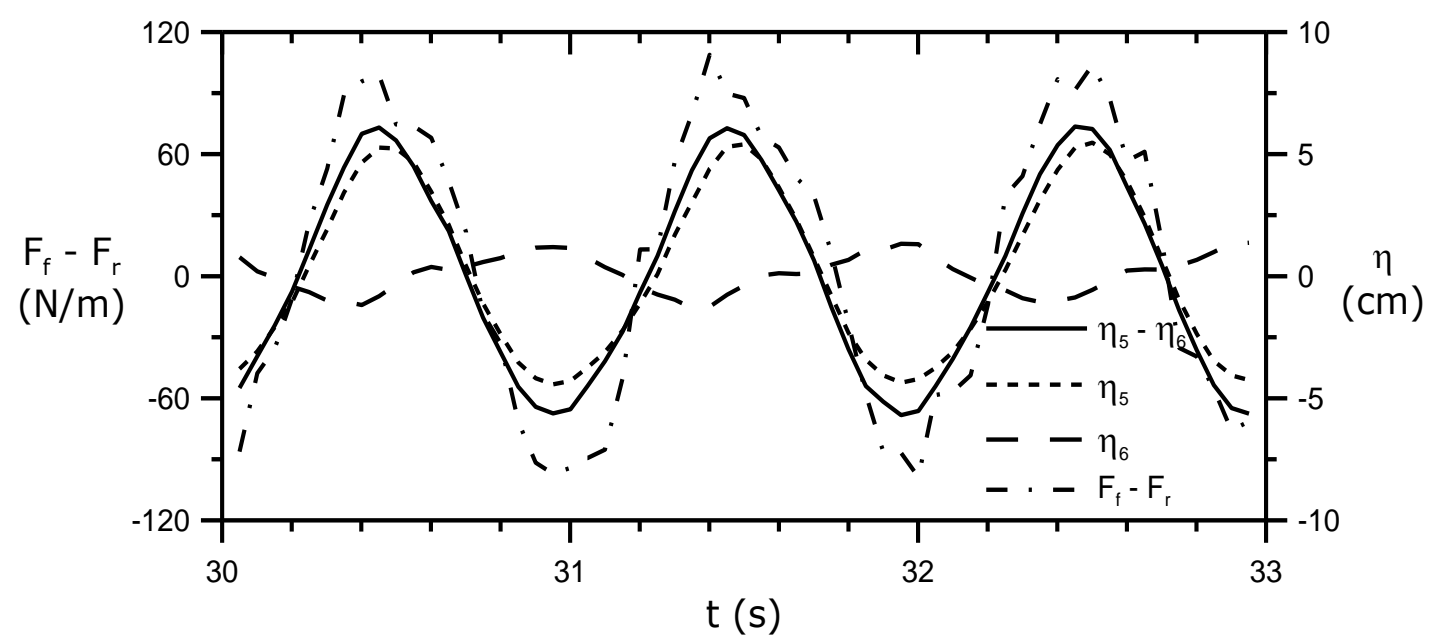

Fig. 3. Temporal variations of water surface elevations at front and rear sides of the first wall, their difference, and total wave force acting on the wall 

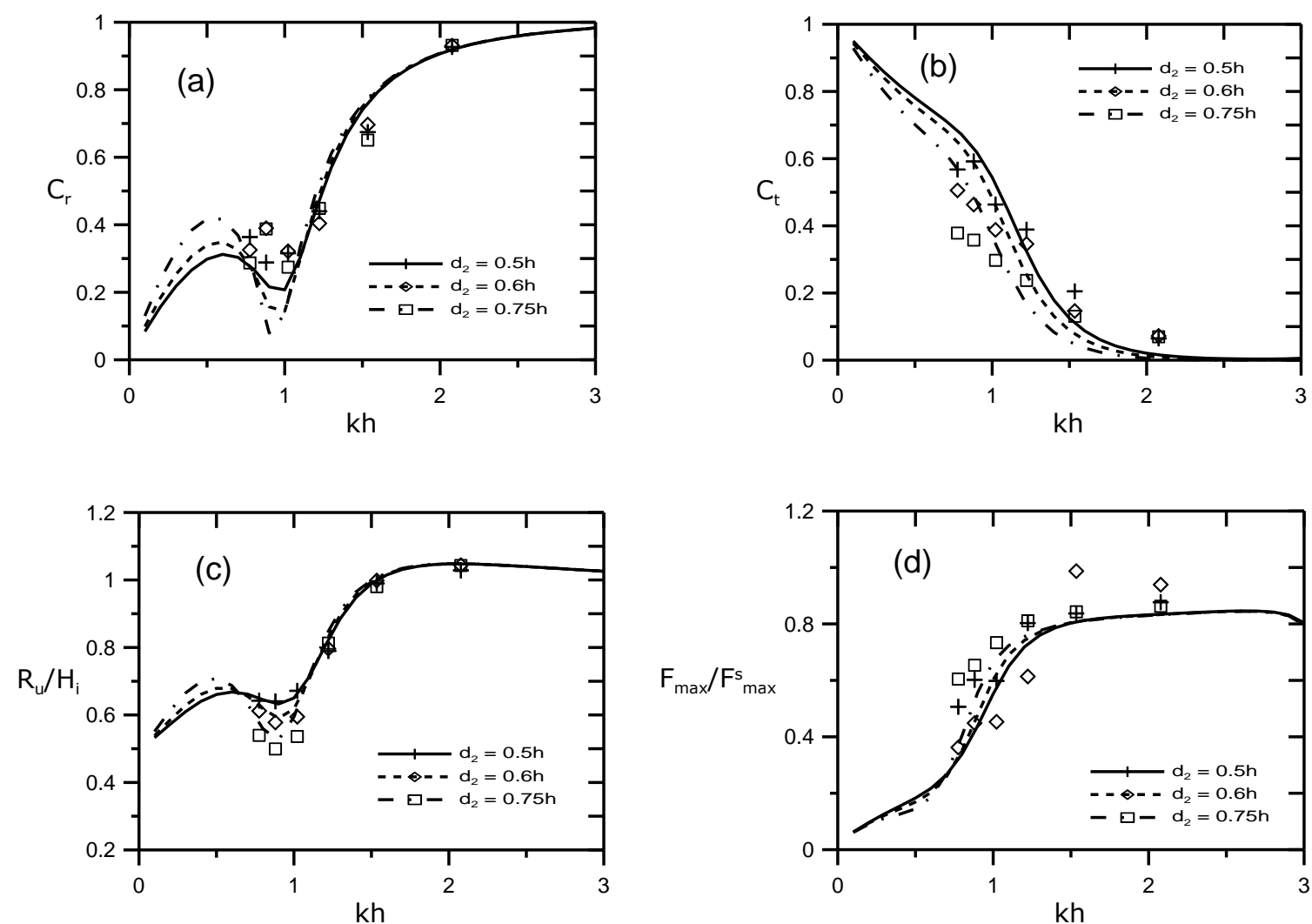

Fig. 4. Comparison of predicted hydrodynamic characteristics with experimental results as function of $k h$ for various drafts of curtain wall of second row: (a) reflection coefficient; (b) transmission coefficient; (c) run-up; and (d) wave force 

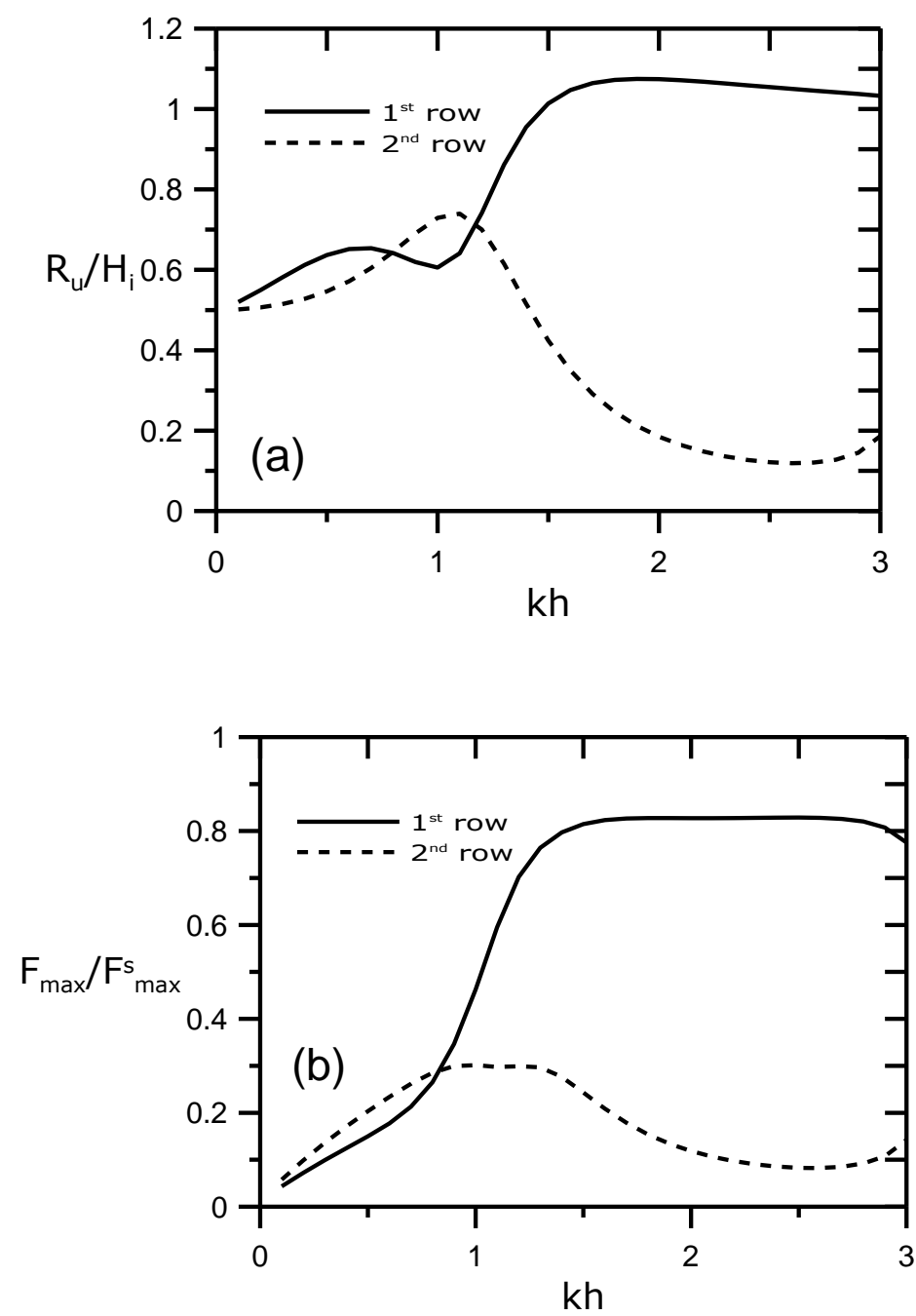

Fig. 5. Comparison of calculated run-up and maximum wave force between first and second rows as function of $k h$ : (a) run-up; and (b) wave force. 

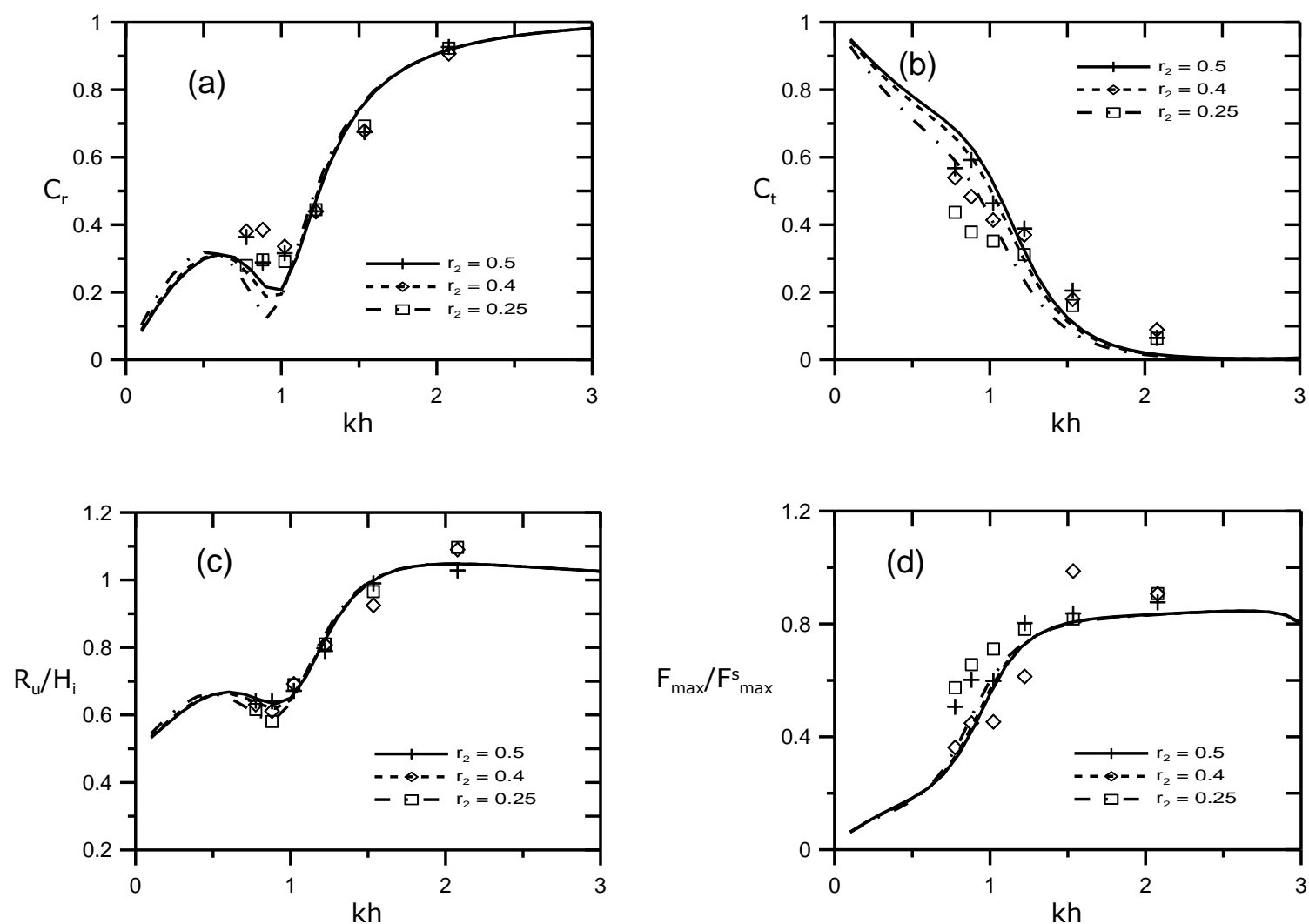

Fig. 6. Comparison of predicted hydrodynamic characteristics with experimental results as function of $k h$ for various porosities between piles of second row: (a) reflection coefficient; (b) transmission coefficient; (c) run-up; and (d) wave force 

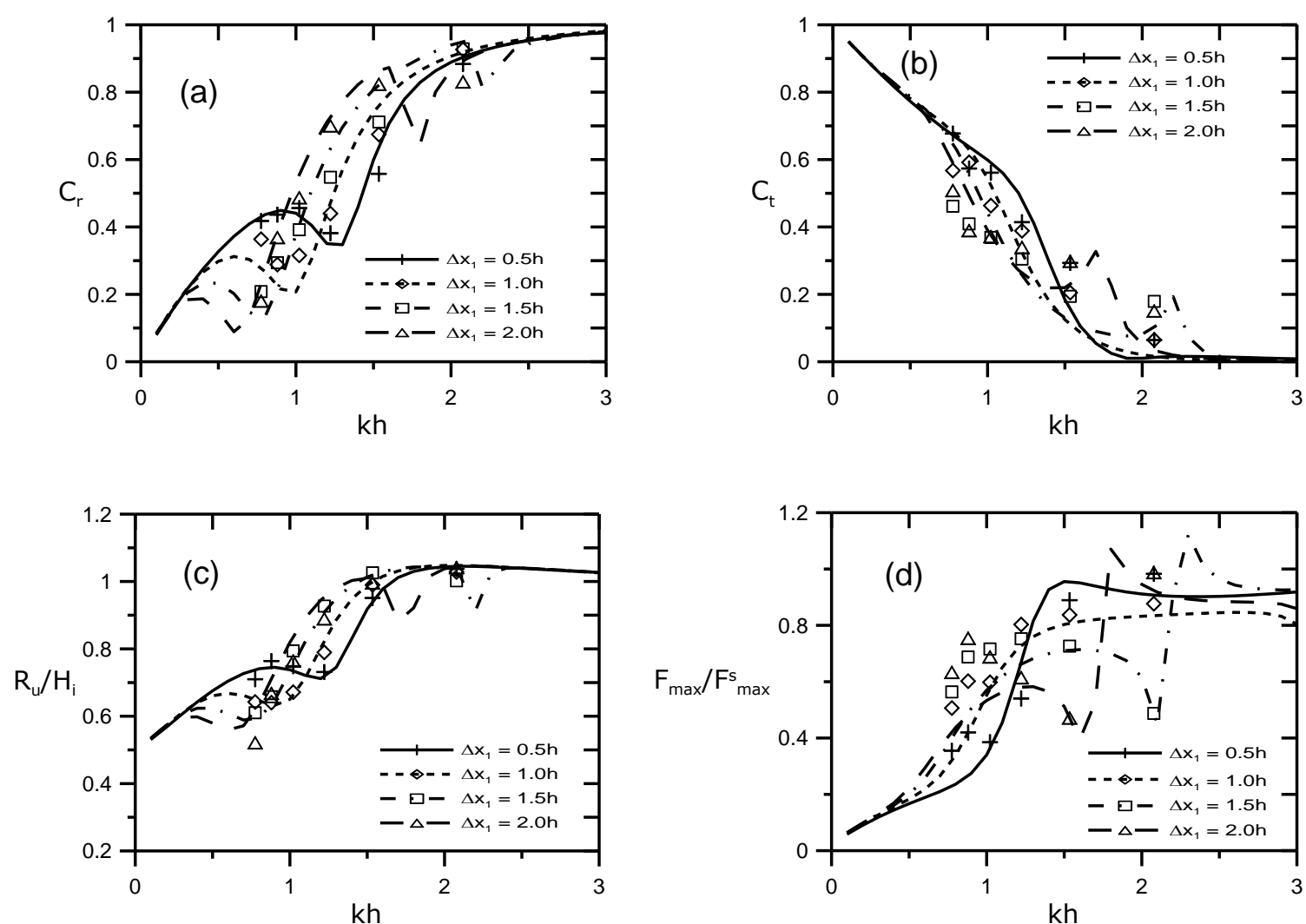

Fig. 7. Comparison of predicted hydrodynamic characteristics with experimental results as function of $k h$ for various distances between first and second rows: (a) reflection coefficient; (b) transmission coefficient; (c) run-up; and (d) wave force 

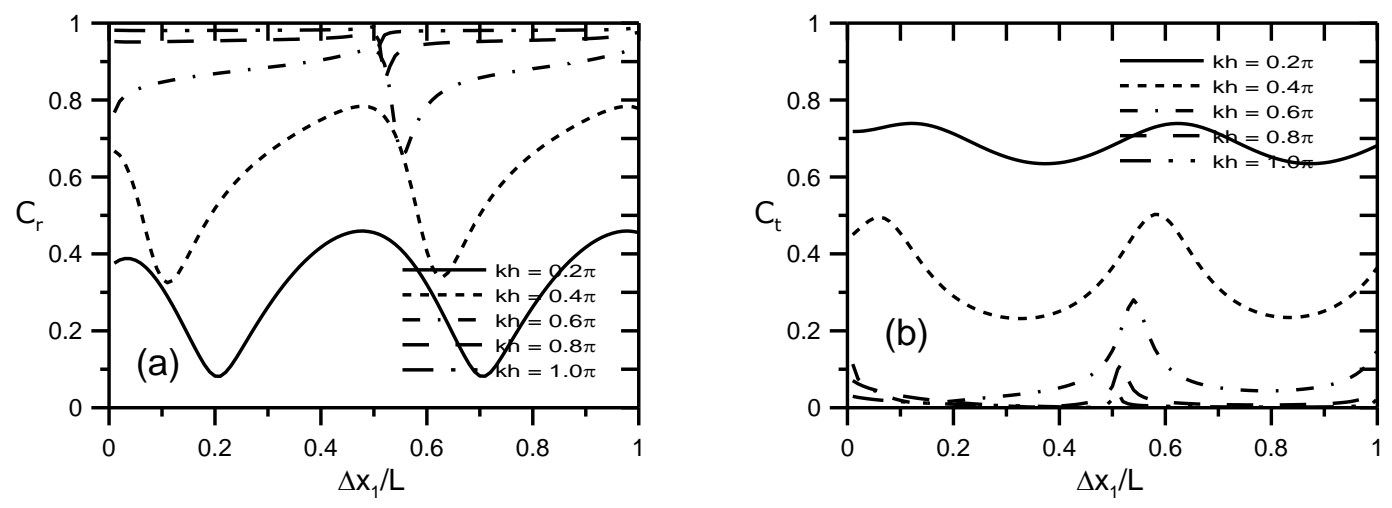

Fig. 8. Predicted reflection and transmission coefficients as function of $\Delta x_{1} / L$ for various relative water depths: (a) reflection coefficient; (b) transmission coefficient 

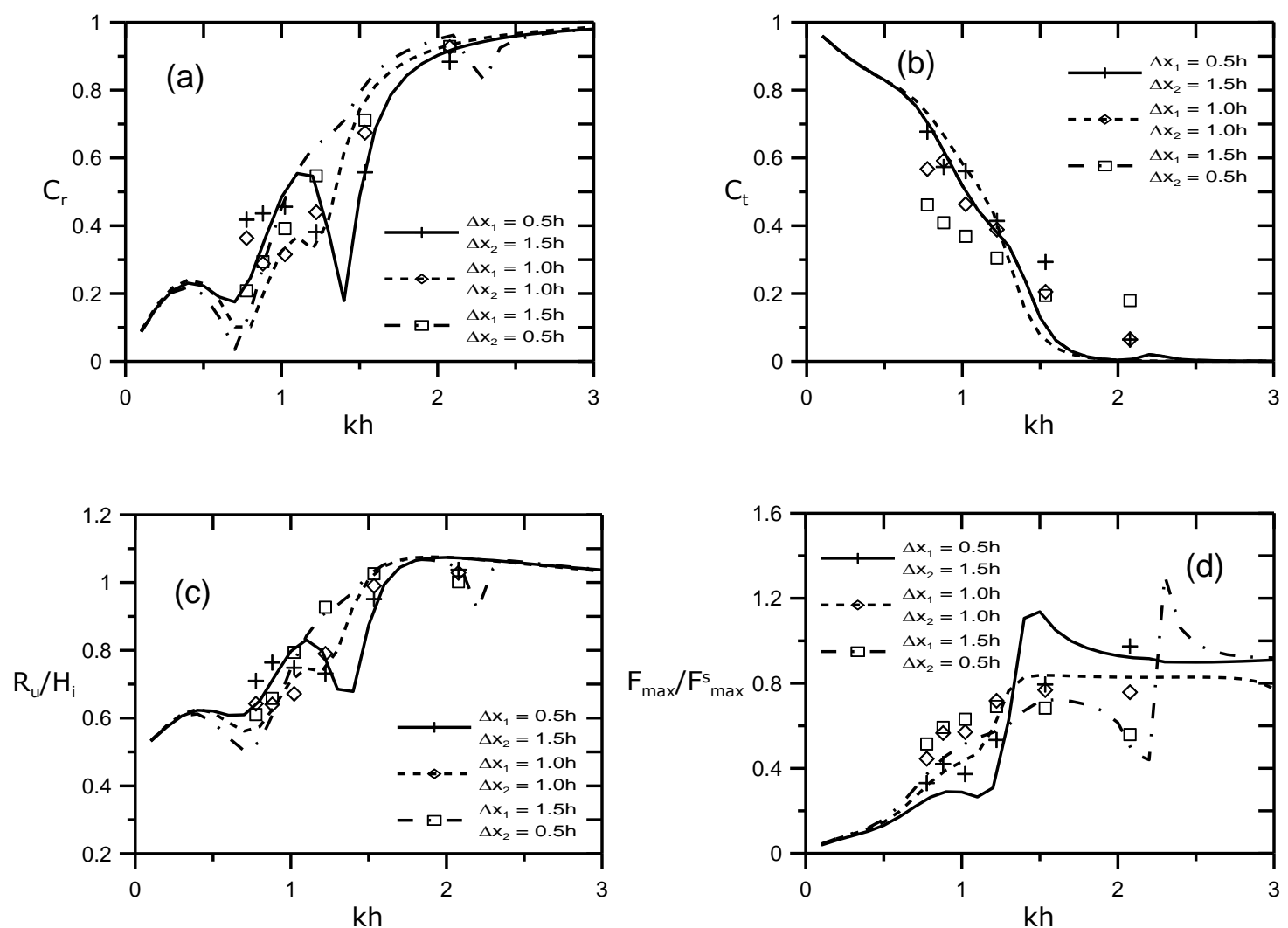

Fig. 9. Comparison of predicted hydrodynamic characteristics with experimental results as function of $k h$ for various distances between rows of triple-row breakwaters: (a) reflection coefficient; (b) transmission coefficient; (c) run-up; and (d) wave force 

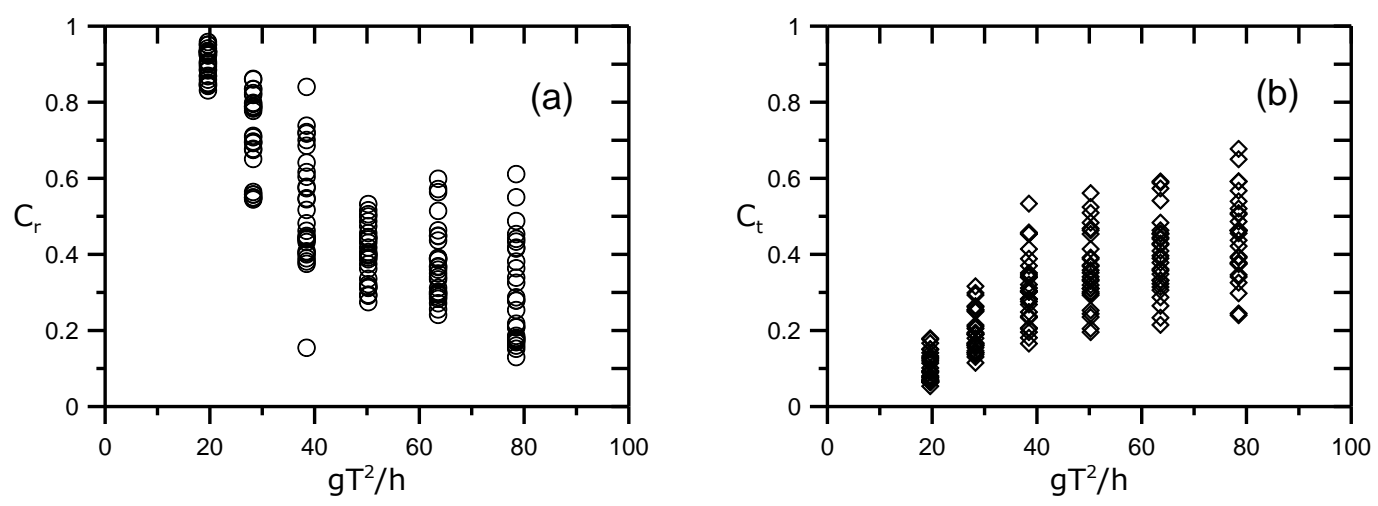

Fig. 10. Measured reflection and transmission coefficients versus $g T^{2} / h$ in prototype breakwaters: (a) reflection coefficient; (b) transmission coefficient 

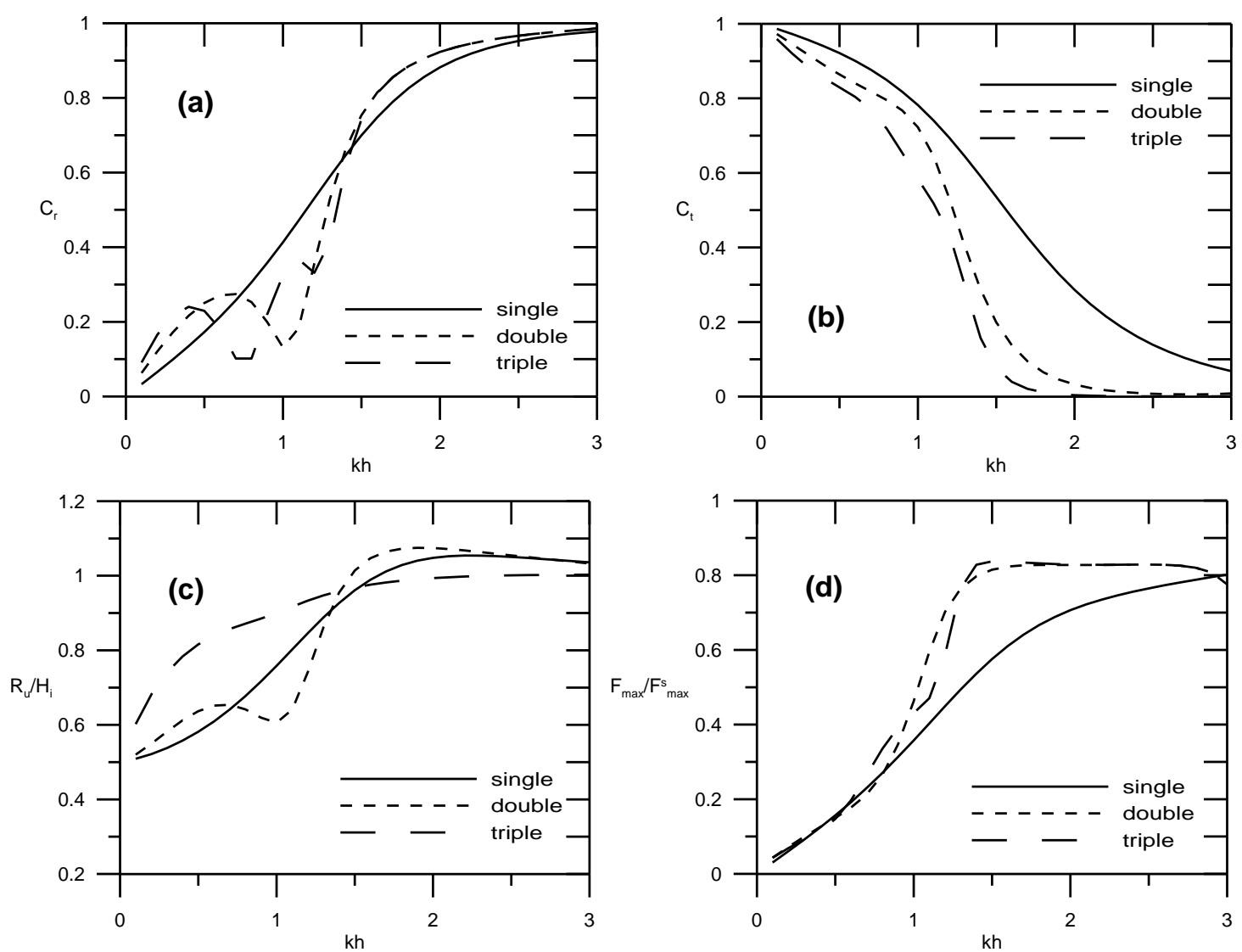

Fig. 11. Comparison of predicted hydrodynamic characteristics between single-row and multiple-rows as function of $k h$ : (a) reflection coefficient; (b) transmission coefficient; (c) run-up; and (d) wave force 

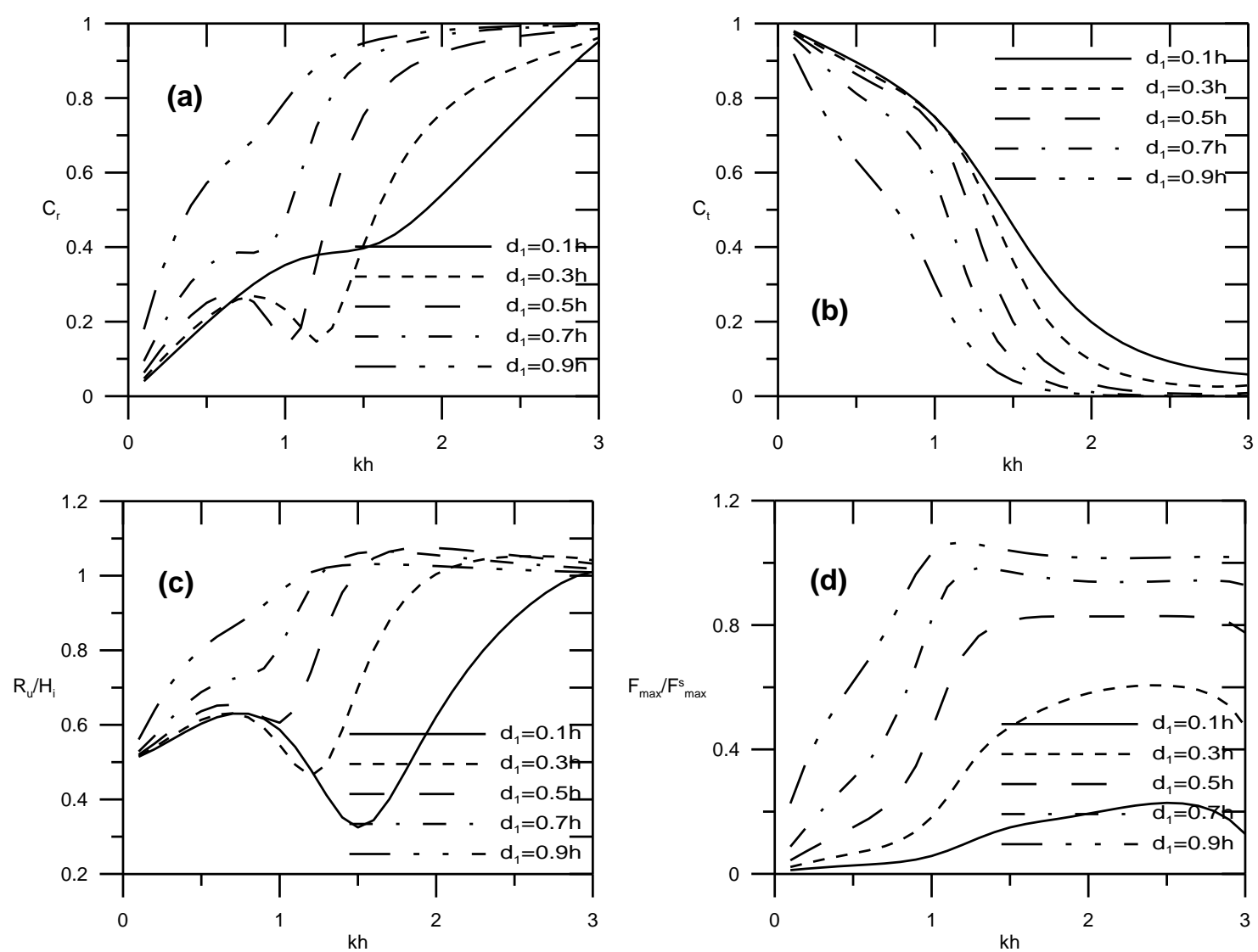

Fig. 12. Variation of hydrodynamic characteristics with change of draft of curtain wall of first row: (a) reflection coefficient; (b) transmission coefficient; (c) run-up; and (d) wave force 

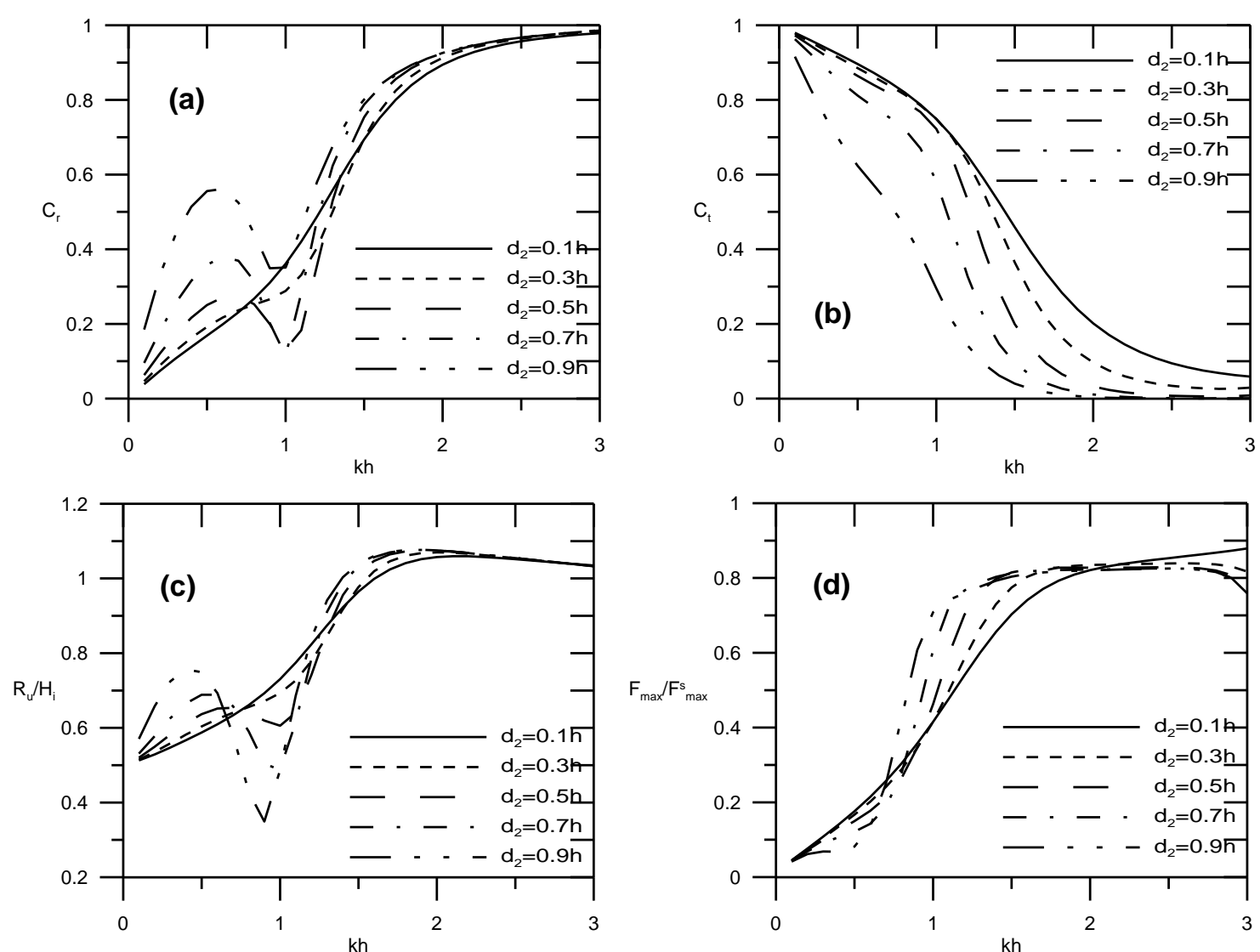

Fig. 13. Variation of hydrodynamic characteristics with change of draft of curtain wall of second row: (a) reflection coefficient; (b) transmission coefficient; (c) run-up; and (d) wave force 

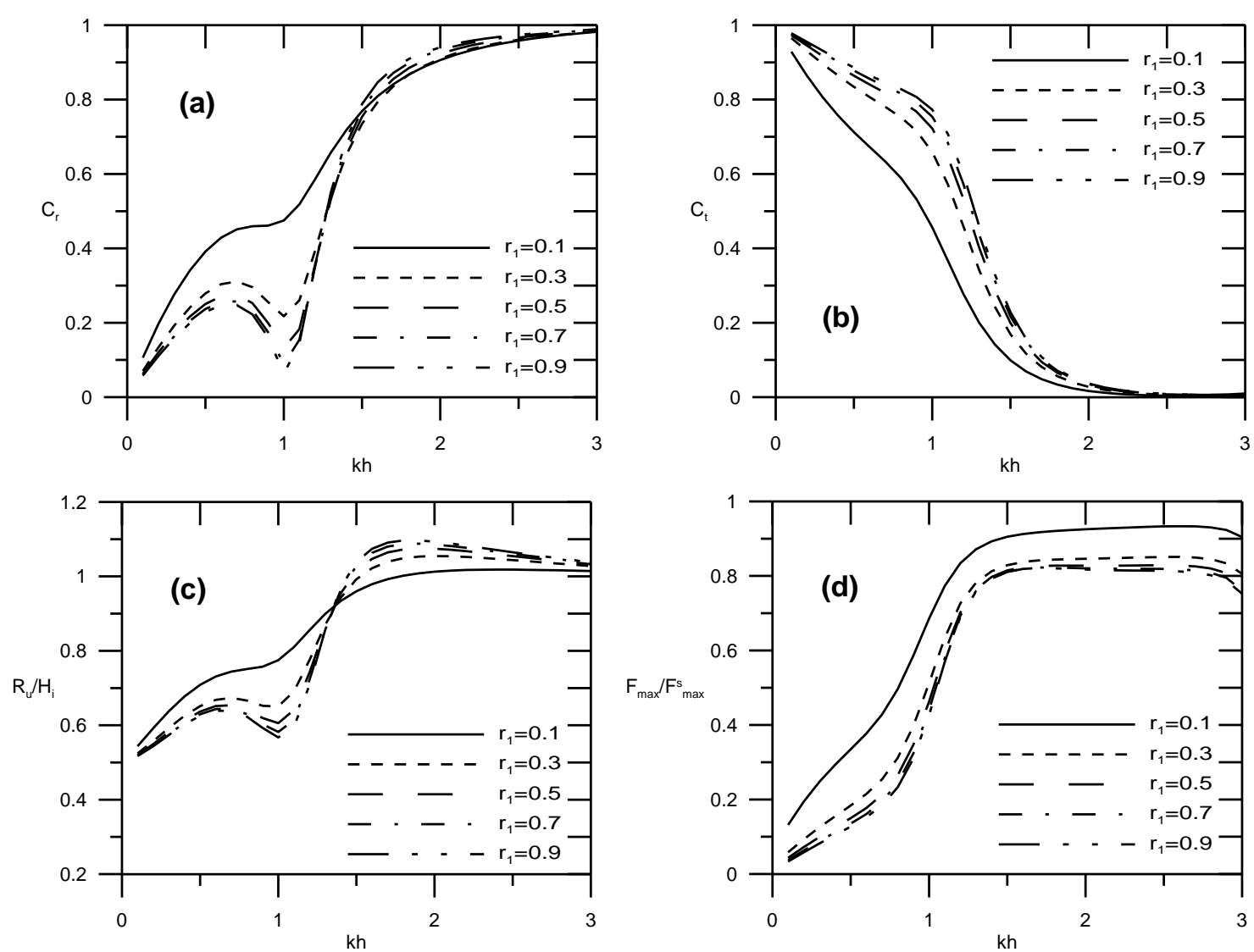

Fig. 14. Variation of hydrodynamic characteristics with change of porosity of first row: (a) reflection coefficient; (b) transmission coefficient; (c) run-up; and (d) wave force 

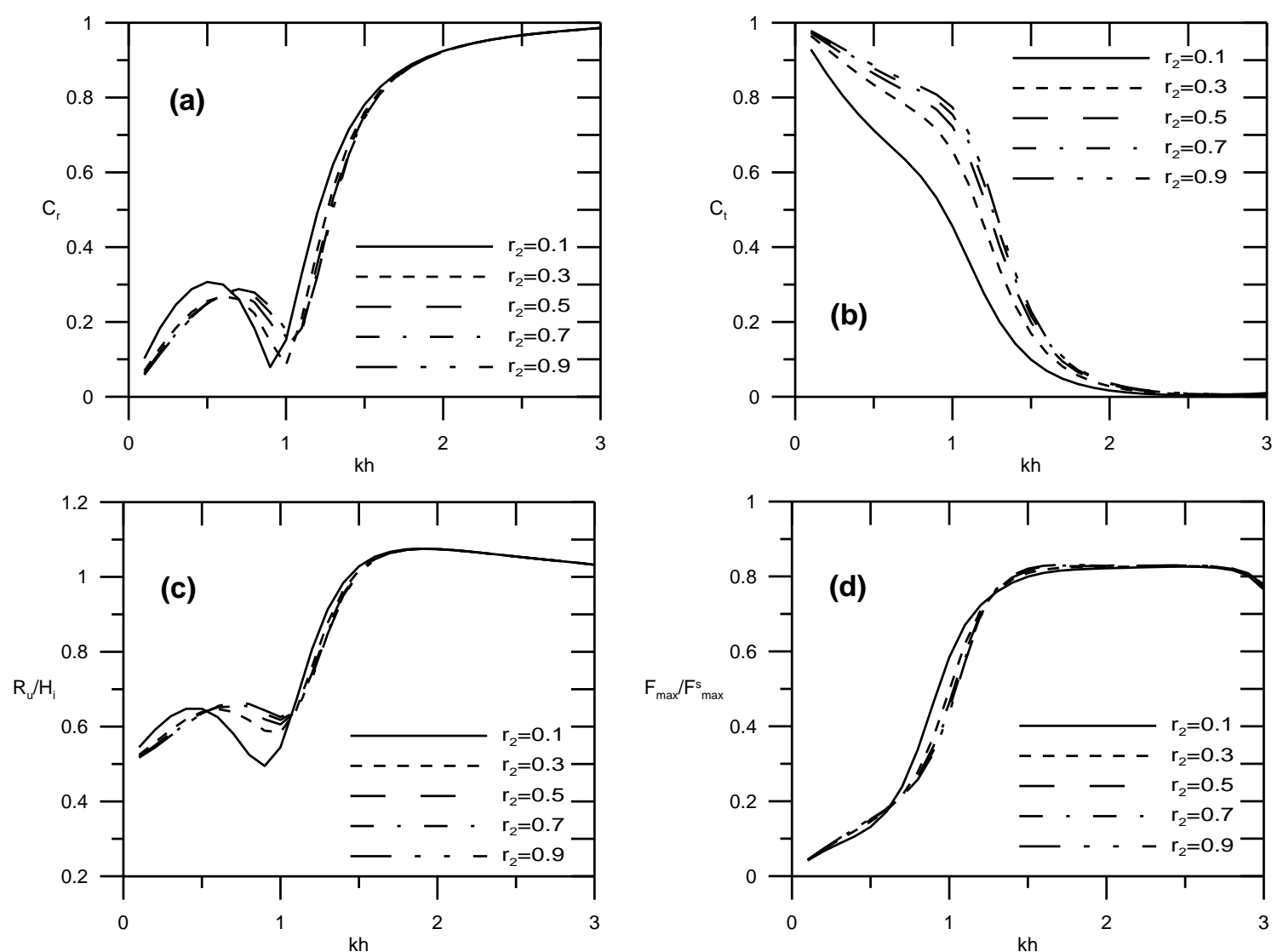

Fig. 15. Variation of hydrodynamic characteristics with change of porosity of second row: (a) reflection coefficient; (b) transmission coefficient; (c) run-up; and (d) wave force 

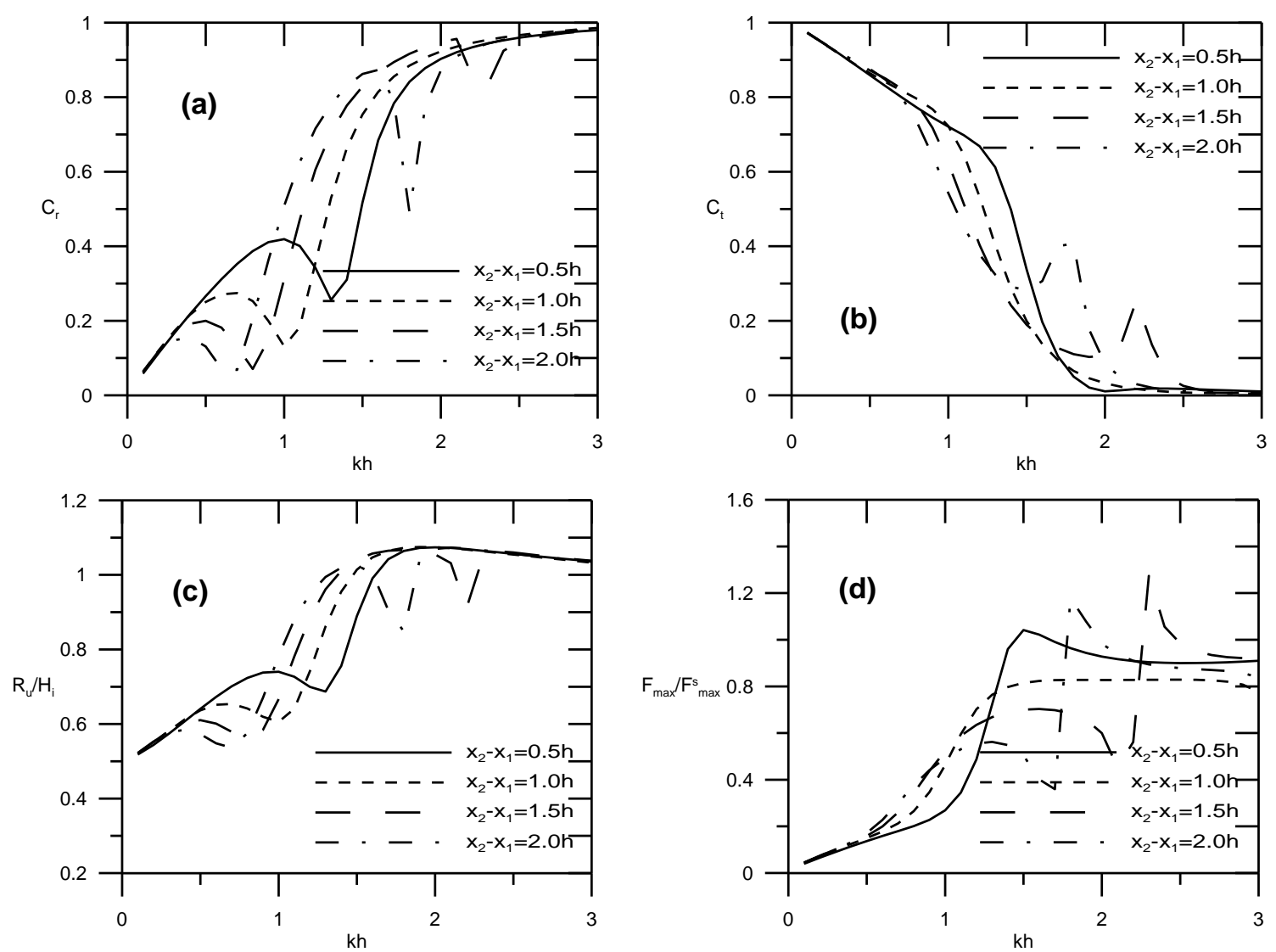

Fig. 16. Variation of hydrodynamic characteristics with change of spacing between rows: (a) reflection coefficient; (b) transmission coefficient; (c) run-up; and (d) wave force 